INPLASY

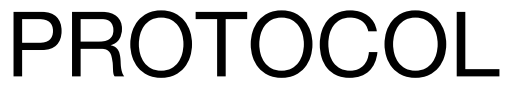

To cite: Guo et al. Efficacy and safety of cutting therapy in the treatment of migraine $-\mathrm{A}$ protocol for systematic review and meta-analysis. Inplasy protocol 2021110029. doi: 10.37766/inplasy2021.11.0029

Received: 08 November 2021

Published: 08 November 2021

Corresponding author:

Yongsheng Huang

gwpdyx2021@163.com

Author Affiliation:

Changchun University of

Chinese Medicine.

Support: None.

Review Stage at time of this submission: The review has not yet started.

Conflicts of interest:

None declared.

\section{Efficacy and safety of cutting therapy in the treatment of migraine - A protocol for systematic review and meta-analysis}

Guo, WP1; Jin, HG2; Wang, YQ33 Zhu, X4; Zhang, GW5; Wang, T6; Fan, $\mathrm{CH}^{7}$; Huang, YS ${ }^{8}$.

Review question / Objective: This study will systematically review and analyze the existing literature on the treatment of migraine with cutting therapy to evaluate its efficacy and safety and to provide objective evidence for clinical practice. Eligibility criteria: All patients with a clinical diagnosis of migraine were included in our study, regardless of whether they had an acute or chronic attack, a first attack or a relapse, and regardless of their gender, age, race, belief, region, economic status and education; the study type was an RCT; the control group were conventional Western medicine treatment (e.g., analgesia, vasodilation), placebo therapy, or psychotherapy. The experimental group was given cutting therapy. Exclusion criteria were as follows: repeated published papers; retrospective studies on cutting treatment of migraine; agreements, conference papers, abstracts, nonfull text, and personal reports on experiences with cutting treatment for migraine.

INPLASY registration number: This protocol was registered with the International Platform of Registered Systematic Review and Meta-Analysis Protocols (INPLASY) on 08 November 2021 and was last updated on 08 November 2021 (registration number INPLASY2021110029).

\section{INTRODUCTION}

Review question / Objective: This study will systematically review and analyze the existing literature on the treatment of migraine with cutting therapy to evaluate its efficacy and safety and to provide objective evidence for clinical practice.

Condition being studied: In today's society, migraine has become a common and frequently occurring clinical disease. A survey shows that more than 1 billion 
people suffer from migraine every year, and there is no difference in the incidence of migraine across various regions. The World Health Organization lists migraine as the sixth most common disabling disease in the world. Therefore, it is of great significance to actively intervene in migraine attacks and prevent migraine recurrence when possible. The onset of migraine is paroxysmal. When it occurs, the patient experiences severe pain on one or both sides of the head, accompanied by photophobia, aversion to sound, nausea and other symptoms. The common existing treatment methods, such as analgesia and vasodilation, have little effect on migraine and have obvious side effects. Cutting therapy is a treatment method based on the theory of traditional Chinese medicine (TCM) and is guided by meridians and acupoints. As a complementary and alternative medicine, cutting therapy has shown good curative effects on migraine. However, there is still a lack of consensus on the efficacy of cutting therapy in the treatment of migraine.Migraine is a chronic paroxysmal neurovascular disease in which pain on one or both sides of the head is the main manifestation and is accompanied by other neurological manifestations. Clinical practice has shown that cutting therapy as a complementary alternative medicine can play a role in relieving migraine attacks. However, there is no consensus on the efficacy of cutting treatment in the treatment of migraine. The aim of this study was to conduct a meta-analysis to systematically evaluate the efficacy and safety of cutting therapy in the treatment of migraine.

\section{METHODS}

Search strategy: The main databases we searched included PubMed, Web of Science, MEDLINE, Embase, Cochrane Library, the Chinese National Knowledge Infrastructure, the Chinese Science Journal Database, Wanfang Data, and the Chinese Biomedical Literature Database. The search time was from database establishment to November 2021. The content of our search was literature related to the efficacy and safety of cutting therapy in the treatment of migraine, including clinical observations and clinical trials. The keywords searched were "cutting treatment", "TCM cutting treatment", and "migraine". To avoid data loss, we manually searched the references of the articles that met the criteria. We searched only for publications in two languages, Chinese or English, regardless of the quality of the publications.

Participant or population: All patients with a clinical diagnosis of migraine were included in our study, regardless of whether they had an acute or chronic attack, a first attack or a relapse, and regardless of their gender, age, race, belief, region, economic status and education.

Intervention: The experimental group was given cutting therapy.

Comparator: The control group were conventional Western medicine treatment (e.g., analgesia, vasodilation), placebo therapy, or psychotherapy.

Study designs to be included: All randomized controlled trials of cutting therapy for migraine were included in our study, did not consider the blinding method or whether the allocation was concealed in the research reported by the included articles. Our study was not limited by publication year and region, but the language of the literature was limited to Chinese and English.

Eligibility criteria: All patients with a clinical diagnosis of migraine were included in our study, regardless of whether they had an acute or chronic attack, a first attack or a relapse, and regardless of their gender, age, race, belief, region, economic status and education; the study type was an RCT; the control group were conventional Western medicine treatment (e.g., analgesia, vasodilation), placebo therapy, or psychotherapy. The experimental group was given cutting therapy. Exclusion criteria were as follows: repeated published papers; retrospective studies on cutting treatment of migraine; agreements, conference papers, abstracts, non-full text, 
and personal reports on experiences with cutting treatment for migraine.

Information sources: Databases were searched for relevant literature. MEDLINE, Embase, Cochrane Library, the Chinese National Knowledge Infrastructure, the Chinese Science Journal Database, Wanfang Data, and the Chinese Biomedical Literature Database. The search was conducted from inception to November 2021.

Main outcome(s): The primary outcome measures were headache symptom score and headache severity visual analog scale (VAS) score.

Additional outcome(s): The secondary outcome measures were sleep condition, diet, and mental health status.

Data management: We will perform this process using consistent data extraction criteria. This process will also be performed independently by 2 researchers, and in case of inconsistencies in the content-extraction process, discussion with a third researcher will be required. The information extracted for this study mainly will include the basic information of the included study, the basic information of the participants, the intervention methods for migraine, and the outcome indicators.

Quality assessment / Risk of bias analysis: The risk of bias for each study will be assessed with the Cochrane Collaboration's tool, and this process will be performed independently by 2 researchers. When the two researchers disagree, a third researcher will make the final decision. The main areas we will assess include randomized sequence generation; allocation sequence concealment; blinding of participants and personnel; blinding of outcome assessment; incomplete outcome data; selective outcome reporting; and other biases. Upon completion of the assessment, each assessed area will be classified as having a low, high or unclear risk of deviation.
Strategy of data synthesis: This study will use Review Manager Version 5.4 software (The Cochrane Collaboration, Copenhagen, Denmark) for meta-analysis. The effects of continuous variables will be expressed as the mean difference (MD) or standardized mean difference. The efficacy of binary variables will be calculated using the hypothetical risk ratio (RR) or odds ratio (OR), setting $95 \%$ as the confidence interval $(95 \% \mathrm{Cl})$. Heterogeneity will be determined with the 12 test. When $12 \leq 50 \%$ and $P>.1$, this indicates that our study is uniform, and the fixed effect model will be selected for meta-analysis. When $12>50 \%$ and $P<.1$, this indicates that the difference is statistically significant, and a randomeffects model will be used.

Subgroup analysis: Subgroup analysis will be performed if data are available and sufficient, such as different intervention time, and different stages of migraine.

Sensitivity analysis: To judge the robustness and stability of the review results, we will conduct a sensitivity analysis. Through sensitivity analysis, we will remove low-quality studies with small sample sizes, high risk of bias, or missing data.

Language: We searched only for publications in two languages, Chinese or English.

\section{Country(ies) involved: China.}

Other relevant information: In the event that relevant data is missing, we will contact the authors by e-mail or telephone. When the author cannot be contacted or if accurate data cannot be obtained after contacting the author, the study will be excluded. Our study involves neither patient recruitment nor animal experimentation and therefore dose not require ethics committee approval.

Keywords: migraine; cutting therapy; metaanalysis; protocol; systematic review.

Contributions of each author:

Author 1 - Wenping Guo. 
Email: 540583116@qq.com Author 2 - Hongguang Jin. Email: 7420465@qq.com Author 3 - Yiqiang Wang. Email: 105993899@qq.com Author 4 - Xing Zhu. Email: 1501625356@qq.com Author 5 - Guanwei Zhang. Email: 994524495@qq.com Author 6 - Te Wang.

Email: 1259143977@qq.com Author 7 - Chunhui Fan.

Email: 1805618839@qq.com Author 8 - Yongsheng Huang.

Email: gwpdyx2021@163.com 